PlastOx 2007 (2009) 57-70

(C) EDP Sciences, 2009

DOI: $10.1051 /$ ptox/2009006

\title{
Physico-chimie des joints de grains : ségrégation - précipitation
}

\author{
L. Priester \\ ICMPE/CNRS, 15 rue Georges Urbain, 94407 Vitry-sur-Seine, France \\ e-mail : louisette.priester@glvt-cnrs.fr
}

\begin{abstract}
Résumé. La densité de défauts ponctuels, lacunes et atomes en insertion ou en substitution, éléments d'alliages ou impuretés, est généralement plus grande aux joints de grains qu'en matrice, hormis pour la macle cohérente $\Sigma 3\{111\}$. Le phénomène de ségrégation intergranulaire affecte non seulement la structure et l'énergie d'un joint de grains mais aussi ses défauts et leurs comportements. Les différences de chimie entre joints peuvent gommer totalement leurs différences géométriques. Étroitement dépendante de la ségrégation, et difficile à différencier de celle-ci à ses tous débuts, la précipitation préférentielle d'une seconde phase aux joints de grains d'une phase initiale (ou phase-mère) joue également un rôle important sur les propriétés d'emploi des matériaux.
\end{abstract}

\section{SÉGRÉGATION INTERGRANULAIRE}

La ségrégation est un phénomène d'enrichissement local en soluté (ajout contrôlé ou impureté) résultant d'une redistribution de celui-ci entre le cristal et certains sites fautés du réseau cristallin (dislocations, surfaces, joints de grains) lors d'un traitement thermique ou thermomécanique. Cette interaction entre défauts ponctuels et défauts linéaires ou planaires entraîne une diminution de l'enthalpie libre du système et une relaxation des défauts. Pour tout matériau polycristallin, les conséquences de la ségrégation interfaciale sur les propriétés structurelles et fonctionnelles sont considérables : fragilité ou consolidation, corrosion intergranulaire, glissement intergranulaire, modification des propriétés électriques...

On distingue généralement deux types de ségrégation selon les forces motrices mises en jeu :

- La ségrégation d'équilibre est gouvernée par les paramètres du système (joint/cristaux) à l'équilibre et non par son histoire. Seul le cœur du joint est fortement affecté par la ségrégation (1 à 2 distances interatomiques)

- La ségrégation hors d'équilibre est généralement due aux gradients thermiques de concentration en lacunes qui apparaissent lors de changements brusques de température. Elle résulte le plus souvent d'une trempe ou d'effets d'irradiation par les neutrons ou encore d'une déformation à grande vitesse. La ségrégation hors d'équilibre affecte une zone relativement large de part et d'autre du joint de grains, pouvant atteindre quelques microns.

On considère essentiellement la ségrégation d'équilibre qui dépend fortement de la structure du joint de grains et qui est mieux connue bien que plus difficile à observer. On met l'accent sur l'anisotropie de la ségrégation d'équilibre d'un joint de grains à l'autre et d'un site à l'autre d'un même joint. Le rôle des défauts de structure des joints de grains dans la ségrégation intergranulaire est aussi abordé. La ségrégation hors équilibre est décrite plus succinctement bien que ce soit un phénomène plus important pratiquement que la ségrégation d'équilibre, mais son approche théorique est moins avancée.

\subsection{Forces motrices de la ségrégation d'équilibre}

Les forces motrices sont soit d'origine élastique, soit d'origine électronique. La considération des premières est souvent suffisante pour comprendre l'interaction et pour obtenir des énergies de ségrégation avec une bonne approximation. On distingue deux types d'interactions élastiques les unes 
sont liées aux différences de taille (effet stérique), les autres aux différences de modules d'élasticité. Dans le premier cas, l'énergie d'interaction pour la ségrégation est égale à la diminution de l'énergie de déformation élastique qui résulte du fait qu'un atome trop gros (ou trop petit) pour un site du cristal trouve un site dans le joint de grains qui lui est mieux adapté. Dans le second cas, l'atome de soluté est considéré comme une inhomogénéité élastique, les effets sont généralement moins prononcés que ceux liés à la taille.

Les effets électroniques sont plus ou moins forts selon les différences d'électronégativité entre soluté et solvant, le rapport e/a et le caractère angulaire des orbitales de valence. Ils sont faibles lorsque le soluté occupe un site dans le joint similaire à un site dans le cristal. Ils sont forts lorsqu'il y a formation de nouvelles liaisons, le site dans le joint diffère du site en matrice conduisant à une nouvelle densité d'états électroniques à l'interface.

\subsection{Brefs rappels sur la thermodynamique de la ségrégation d'équilibre}

Dans une solution idéale suffisamment diluée, l'enrichissement en élément soluté du joint de grains suit l'expression classique de Gibbs :

$$
\Gamma=-\frac{1}{\mathrm{RT}}(\partial \gamma / \partial \ln X)_{\mathrm{T}, \mathrm{V}}
$$

Cette expression relie $\Gamma$, l'excès de moles $\mathrm{n}$ de l'espèce soluté par unité de surface du joint de grains, à la variation de l'énergie intergranulaire $\gamma\left(\mathrm{en} \mathrm{J} / \mathrm{m}^{2}\right)$ en fonction de la fraction molaire de soluté en matrice $\mathrm{X}$. R est la constante des gaz parfaits. La pente de la courbe $(\partial \gamma / \partial \ln X)$ pour $\mathrm{X}=0$ indique la tendance à la ségrégation du soluté aux joints de grains.

Pour une solution binaire régulière et en faisant l'hypothèse que le soluté et le solvant occupent le même volume molaire partiel, l'équation qui décrit la ségrégation aux joints de grains en fonction de la composition et de la température de la solution solide a la forme suivante [1] :

$$
\frac{\mathrm{X}^{\mathrm{J}}}{1-\mathrm{X}^{\mathrm{J}}}=\frac{\mathrm{X}}{1-\mathrm{X}} \exp (-\Delta \mathrm{G} / \mathrm{RT})
$$

$\mathrm{X}^{\mathrm{J}}$ est la fraction molaire de soluté au joint et $\Delta \mathrm{G}$ est l'enthalpie de ségrégation du soluté au joint.

L'équation (2) été déclinée sous différentes formes selon diverses hypothèses. En considérant la solubilité limite $\mathrm{X}_{\max }$. du soluté en matrice et pour des niveaux faibles d'enrichissement $\mathrm{X}^{\mathrm{J}} \ll \mathrm{X}_{\max }^{\mathrm{J}}$, on a :

$$
\frac{\mathrm{X}^{\mathrm{J}}}{\mathrm{X}_{\max }^{\mathrm{J}}}=\frac{1}{\mathrm{X}_{\max }} \exp (-\Delta \mathrm{G} / \mathrm{RT})
$$

Le premier membre de cette équation est le coefficient d'enrichissement intergranulaire $\beta$. Si l'élément ségrégé s'étend à saturation sur une monocouche dans le joint $\left(\mathrm{X}_{\max }^{\mathrm{J}}=1\right), \beta$ evient le rapport de la concentration du soluté au joint sur sa concentration en matrice : $\beta=\mathrm{X}^{\mathrm{J}} / \mathrm{X}$. A partir de l'équation [3], $\beta$ étant inversement proportionnel à la limite de solubilité en matrice du soluté $\mathrm{X}_{\max }$, on peut prédire les valeurs de $\beta$ pour divers solutés de $\mathrm{X}_{\max }$ (Fig. 1) [2]. Souvent, les atomes de solutés ne remplissent pas tous les sites possibles dans le joint, on définit alors un taux de recouvrement $\theta^{\mathrm{J}}=\mathrm{X}^{\mathrm{J}} / \mathrm{X}_{\max }^{\mathrm{J}}$.

\subsection{Ségrégation et paramètres géométriques des joints de grains}

Des diagrammes ternaires de ségrégation intergranulaire dans le fer $\alpha$ représentent la variation de l'enthalpie de ségrégation avec la solubilité maximale $\mathrm{X}_{\max }$ et avec un des paramètres géométriques du joint [3]. Ils ont été établis pour des joints symétriques de flexion [100], le paramètre variable étant l'angle de désorientation $\theta$ et le plan médian des joints étant fixé (Fig. 2a) ou pour une désorientation fixe, la variable étant l'angle $\varphi$ entre l'orientation symétrique (031) et une orientation quelconque 


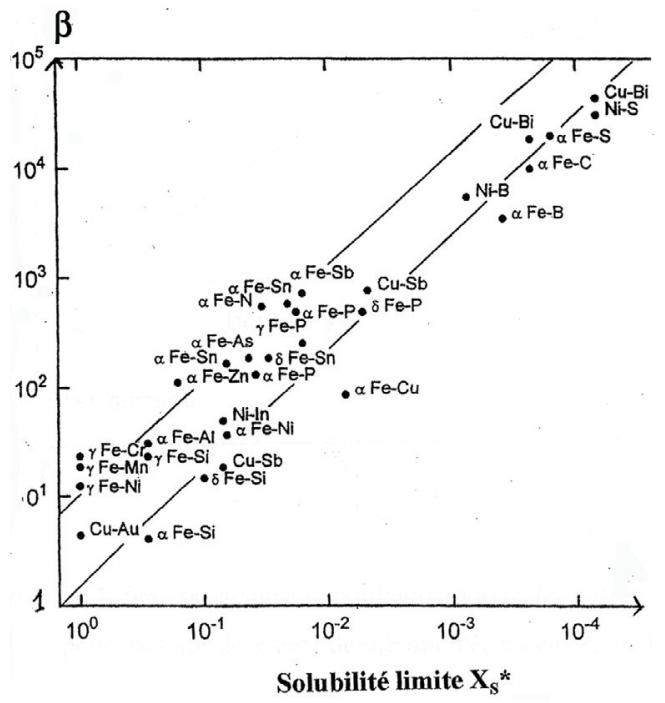

Figure 1. Courbes montrant (en coordonnées logarithmiques) la relation entre le coefficient d'enrichissement $\beta$ et la solubilité en matrice de différents solutés dans le fer, le cuivre et le nickel [2].

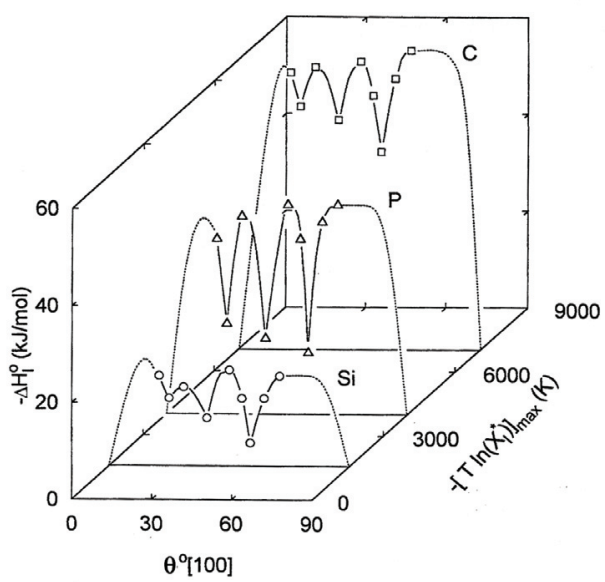

(a)

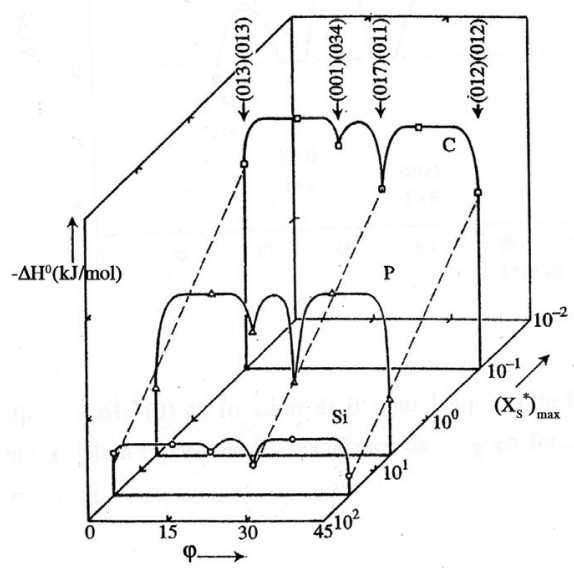

(b)

Figure 2. Variations des enthalpies de ségrégation de $\mathrm{Si}, \mathrm{P}$ et $\mathrm{C}$ dans des joints de grains symétriques de flexion autour de [100] dans le fer en fonction de $X_{\max }$ et en fonction : (a) de la désorientation $\theta$ entre cristaux, (b) de l'inclinaison $\varphi$ du plan dans les joints de grains $\Sigma 536^{\circ} 9$ [100] (voir texte) [3].

du plan du joint de grains (Fig. 2b). On constate que l'anisotropie de l'enthalpie de ségrégation est qualitativement similaire pour les trois solutés, silicium, phosphore et carbone.

Expérimentalement, une attaque électrochimique préférentielle des régions enrichies en soufre dans un polycristal de nickel permet d'estimer le contenu en cet élément d'un joint à l'autre et le long d'un même joint de grains [4]. Les résultats permettent de proposer un critère géométrique de ségrégation basé sur l'espacement moyen $\mathrm{d}_{\mathrm{m}}$ des plans parallèles au plan du joint (Fig. 3). La valeur réduite $\mathrm{d}_{\mathrm{m}} / \mathrm{a}$ (a étant le paramètre de maille du cristal) semble devoir excéder une valeur critique $(\approx 0,150)$ pour que le joint de grains soit peu sensible à la ségrégation. Bien que l'on constate qu'une grande distance 


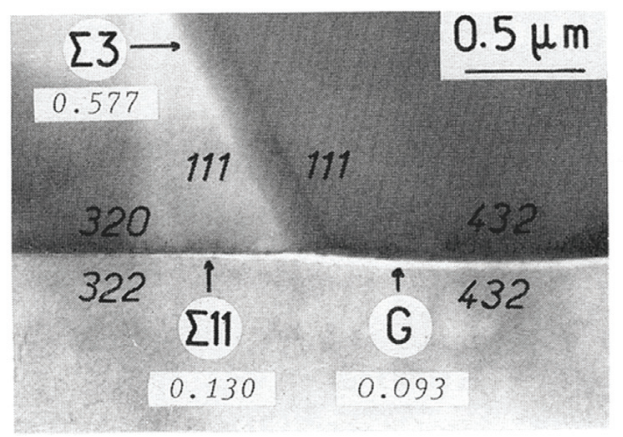

Figure 3. Observation en MET des faciès d'attaque électrochimique de trois joints de grains dans du nickel, attaque associée à la présence de soufre. Pas d'attaque du joint $\Sigma 3\left(\mathrm{~d}_{\mathrm{m}} / a=0,577\right)$, faible attaque du joint $\Sigma 11$ $\left(\mathrm{d}_{\mathrm{m}} / a=0,130\right)$, attaque forte du joint général $\left(\mathrm{d}_{\mathrm{m}} / \mathrm{a}=0,093\right)$ (présence d'un liseré blanc le long du joint)- voir texte [4].

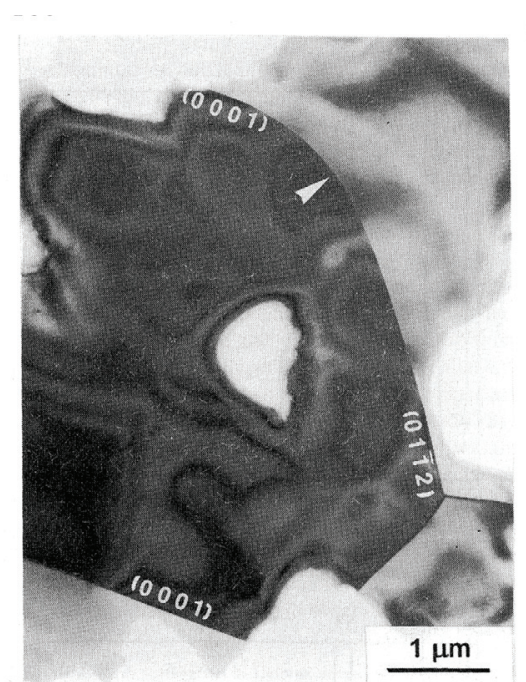

(a)

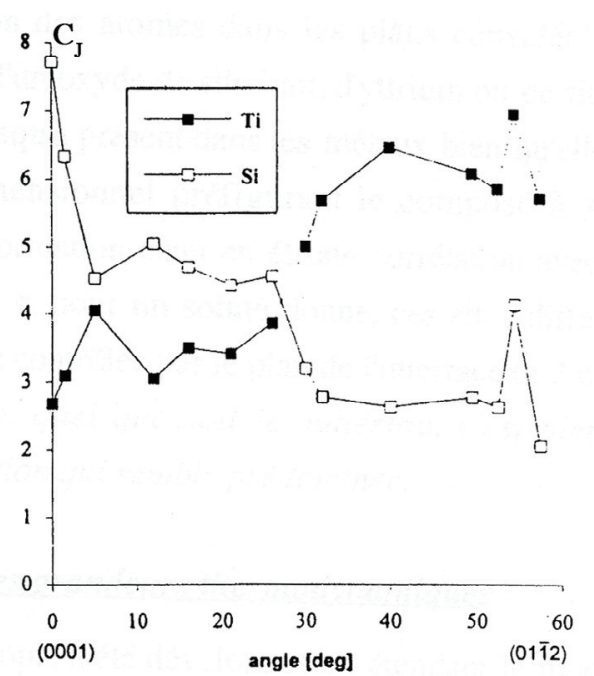

(b)

Figure 4. (a) Image en MET d'un joint de grains courbe dans l'alumine dont le plan passe progressivement de l'orientation basale (0001) à l'orientation rhomboédrique (01-12); (b) Variations des teneurs en titane et en silicium dans le joint en fonction de l'orientation de son plan [5].

interplanaire est souvent associée à un faible niveau de ségrégation dans les joints de flexion, ce critère doit être utilisé avec prudence. D'ailleurs, aucun critère géométrique ne permet de prédire la ségrégation intergranulaire.

L'étude de la ségrégation dans les joints de grains d'une céramique met clairement en évidence le rôle important du plan du joint. Non seulement la quantité, mais aussi la nature de l'élément ségrégé diffère d'un plan de joint à l'autre. Ainsi, dans une alumine polycristalline dopée à l'yttrium contenant par ailleurs du silicium, ce dernier se localise préférentiellement dans un joint de grains dont un des plans est un plan basal (0001) et l'yttrium dans un joint de plan rhomboédrique (01-12). De manière similaire, dans un joint de désorientation donnée d'une alumine commerciale, le titane est majoritaire dans la partie du joint de plan rhomboédrique et le silicium prédomine largement sur le plan basal (Fig. 4) [5]. 


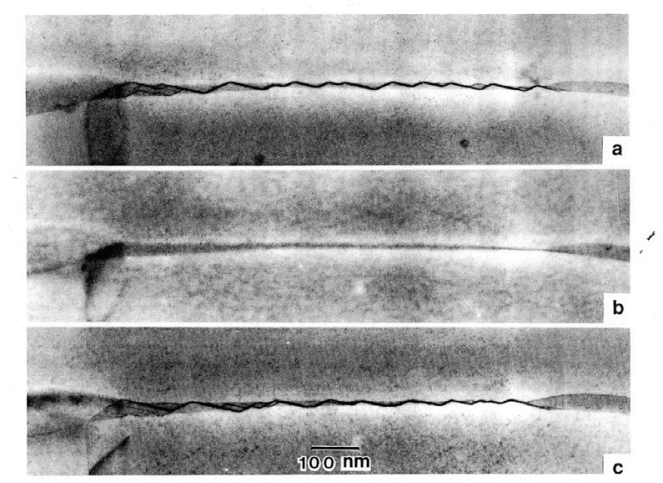

Figure 5. (a) Présence de facettes dans un joint de grains du cuivre contenant du bismuth ; (b) le même joint sans facettes après retrait du bismuth sous l'effet de la température ; (c) les facettes réapparaissent après réintroduction du bismuth [6].
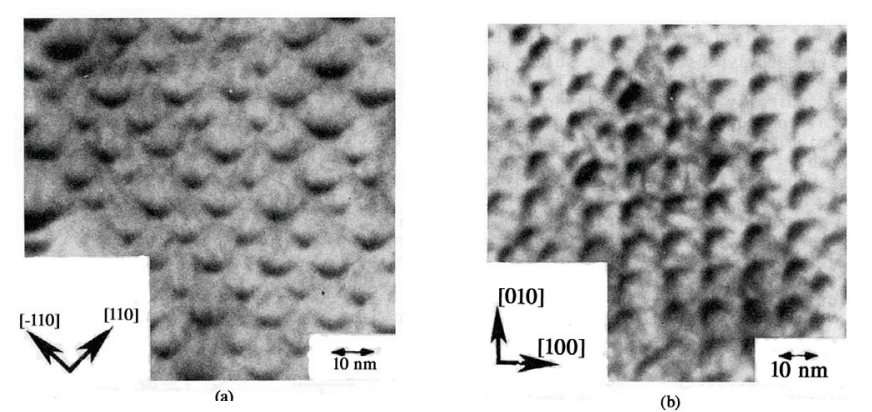

Figure 6. Micrographies électroniques montrant des grilles de dislocations vis dans un joint de torsion $\theta=1^{\circ} 5$ [001] : (a) dans le fer pur, les dislocations sont parallèles aux directions $\langle 110\rangle$ (b) dans Fe- $0,18 \%$ at. Au, les dislocations sont parallèles aux directions $\langle 100\rangle$ [7].

La formation de facettes est une transition de phases qui peut être provoquée par un changement de composition du joint de grains. Ainsi la transformation dans le cuivre pur d'un joint plan en joint à facettes est obtenue sous l'effet de la ségrégation du bismuth, le phénomène étant réversible (Fig. 5) [6].

En conclusion, simulations et expériences montrent une forte relation réciproque entre la ségrégation intergranulaire et le plan du joint qui détermine la structure fine de l'interface.

\subsection{Ségrégation et structures des joints de grains}

La relation réciproque entre la ségrégation et la structure de dislocations d'un joint reste peu analysée alors que les simulations de la ségrégation basées sur la description atomique du joint sont nombreuses.

\subsubsection{Ségrégation intergranulaire et dislocations intrinsèques du joint de grains}

L'attraction qu'exercent les dislocations intrinsèques sur des atomes de soluté et l'influence de ces derniers sur la structure du joint ont fait l'objet de peu d'investigations expérimentales. Cependant, un changement de la structure de dislocations, d'un joint de grains à faible angle sous l'effet d'une ségrégation a été observé en microscopie électronique en transmission dans le cas du système fer - or [7]. 

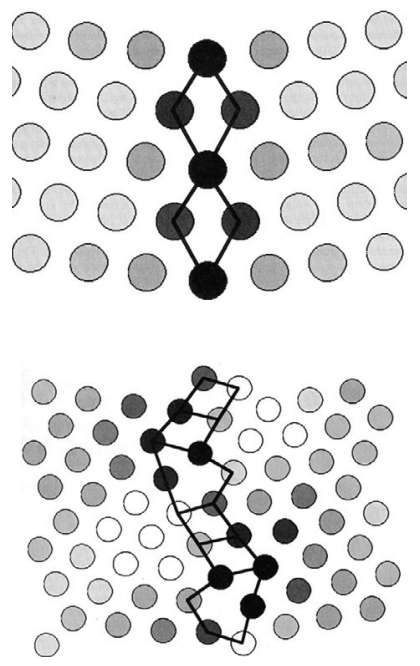

(a)

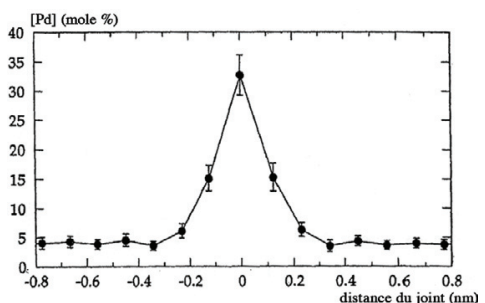

(b)

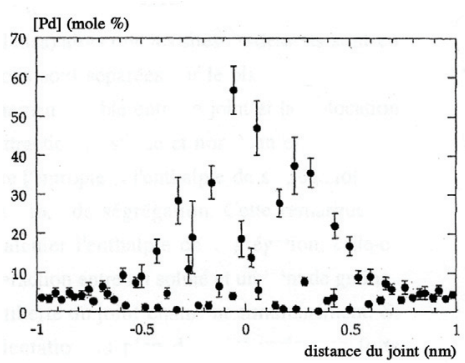

Figure 7. Distribution du palladium site par site (à un site plus sombre correspond une plus forte concentration en palladium) et en fonction de la distance au plan du joint dans les joints de grains du nickel : (a) $\Sigma 11\{113\}$; (b) $\Sigma 33\{441\}[8]$.

L'étude par simulation de dix joints de torsion [001] dans un alliage dilué Ni-Cu montre que dans tous les cas, la ségrégation du cuivre est maximale dans les unités minoritaires associées aux dislocations intrinsèques (voir la partie Structures et défauts). La ségrégation apparaît donc liée à la structure de cœur du joint de grains.

\subsubsection{Ségrégation intergranulaire et structure atomique du joint de grains}

Les simulations de la ségrégation intergranulaire en relation avec la structure atomique du joint ont permis de déterminer l'énergie de ségrégation associée à chaque site du joint de grains, de révéler la grande anisotropie de ségrégation d'un site à l'autre, de prédire la formation d'un composé ordonné intergranulaire et l'intervention de transformations structurales dues à la ségrégation. La figure 7 montre la distribution du palladium, site par site et en fonction de la distance au plan du joint, pour $\Sigma 11$ $\{113\}$ et $\Sigma 33\{441\}$ dans le nickel. L'atome de palladium est nettement plus gros que l'atome de nickel (10\%), mais a le même nombre d'électrons de valence, l'effet élastique est donc largement prédominant. Deux types de sites sont distingués : les sites au cœur du joint concentrent généralement la plus forte concentration en palladium, les sites situés dans la région déformée élastiquement au voisinage du joint contribuent parfois notoirement au niveau de ségrégation totale. La distribution du soluté, homogène et limitée à $0,3 \mathrm{~nm}$ autour du joint $\Sigma 11$, devient hétérogène et étendue autour du joint général $\Sigma 33$ [8].

La figure 8 montre les structures atomiques des joints $\Sigma 11\{113\}$ et $\Sigma 11\{332\}$ et leurs sites possibles de ségrégation dans le système $\mathrm{Ni}(\mathrm{Ag})$ ou $\mathrm{Ag}(\mathrm{Ni}), \mathrm{Ni}$ et $\mathrm{Ag}$ étant soit le soluté (entre parenthèse) soit le solvant. Sont également présentées les variations des énergies de ségrégation en fonction de la distance du site au plan du joint. Pour le joint $\Sigma 11\{113\}$, la concentration en soluté est limitée sur 0,5 nm et varie régulièrement avec la distance au plan du joint. En revanche, pour le joint $\Sigma 11$ \{332\}, l'énergie de ségrégation fluctue de part et d'autre du plan du joint en particulier pour le système $\mathrm{Ni}(\mathrm{Ag})$. La ségrégation de l'atome d'argent plus gros que celui de nickel est favorisé dans le joint $\Sigma 11\{113\}$ par un effet stérique. Inversement le nickel ne ségrége pas dans ce joint de l'argent [9]. 

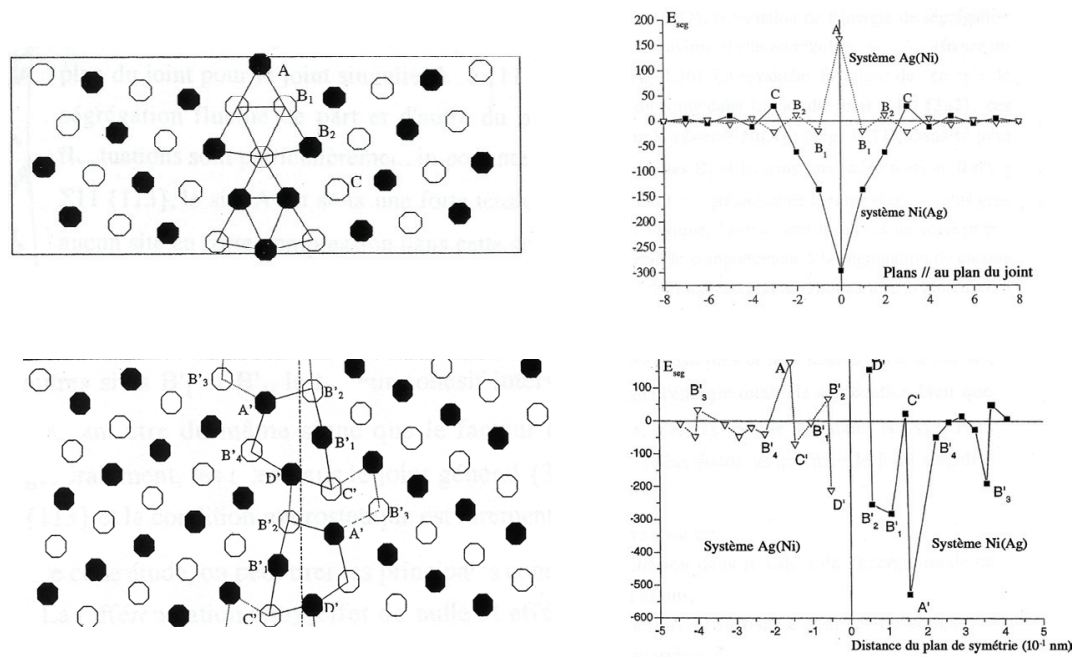

Figure 8. Structure atomique calculée du joint de grains et variations de l'énergie de ségrégation avec la distance au plan, du joint pour : (a) $\Sigma 11\{113\}$; (b) $\Sigma 11\{332\}$, dans ce cas les variations ne sont reportées que d'un côté du joint pour chaque système [9].

Ces simulation montrent bien qu'il y a une forte anisotropie de plan à plan, que la considération seule du facteur stérique est souvent suffisante pour rendre compte du degré de ségrégation par site ; l'effet de cohésion intervient lorsqu'il est relativement important et de signe opposé à l'effet de taille.

Si la structure atomique joue fortement sur la ségrégation à un joint de grains, réciproquement, la ségrégation peut entraîner des changements de structure intergranulaire. Ainsi, les atomes de phosphore ségrégés sous forme d'une monocouche dans les joints de flexion symétriques $\Sigma 5$ (310) et $\Sigma 9$ (114) du fer $\alpha$ provoquent une complète restructuration des joints. Initialement placés en substitution dans les unités structurales, ils occupent ensuite les centres de prismes trigonaux d'atomes de fer nouvellement formés dans les deux types de joints. La configuration obtenue est comparable à celle du phosphore dans le composé tridimensionnel $\mathrm{Fe}_{3} \mathrm{P}$ (Fig. 9) [10].

Une seule technique permet d'observer la ségrégation intergranulaire à l'échelle atomique, c'est la sonde atomique tomographique. La figure 10 présente une image 3D d'un joint général entre deux grains $\gamma^{\prime}$ dans un superalliage N18 révélant nettement la ségrégation du chrome, du molybdène et du bore et la déplétion en aluminium au cœur de joint. Les atomes ségrégés forment un film continu d'épaisseur proche de $1 \mathrm{~nm}$ le long du joint [11].

La ségrégation a également une influence forte sur les structures des joints à l'échelle électronique, en particulier dans les matériaux ioniques et covalents, mais aussi dans les métaux où les éléments ségrégés peuvent consolider ou, à l'inverse, fragiliser le joint. La considération des changements de liaison des atomes dans les unités structurales pourrait permettre d'interpréter ces effets.

\subsection{Ségrégation et défauts de structure du joint de grains}

La ségrégation des atomes de soluté sur les dislocations extrinsèques, à l'instar de leur ségrégation sur les dislocations de matrice, s'explique par l'existence de champs élastiques de déformation et de contrainte à grande distance. Notons qu'il s'agit ici d'une ségrégation d'équilibre bien que les dislocations extrinsèques soient des défauts "hors équilibre" dans le joint de grains. La figure 11 montre un exemple de la surconcentration en yttrium au voisinage immédiat de deux dislocations extrinsèques stabilisées dans un joint de grains général d'alumine [12]. 

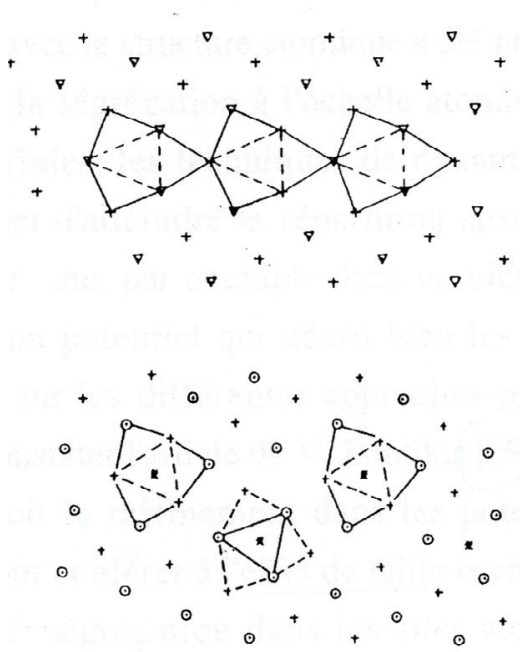

Figure 9. Unités polyédriques d'atomes de fer dans un joint de flexion $\Sigma 5\{310\}$. Les différents symboles indiquent les différentes cotes le long de l'axe $\langle 110\rangle$. Les atomes de phosphore insérés, à raison d'un atome par unité, provoquent un changement de structure (déformation et déplacement des unités initiales). Chaque atome occupe un site interstitiel (étoile) entouré de 3 atomes de fer pour former $\mathrm{Fe}_{3} \mathrm{P}$ [10].
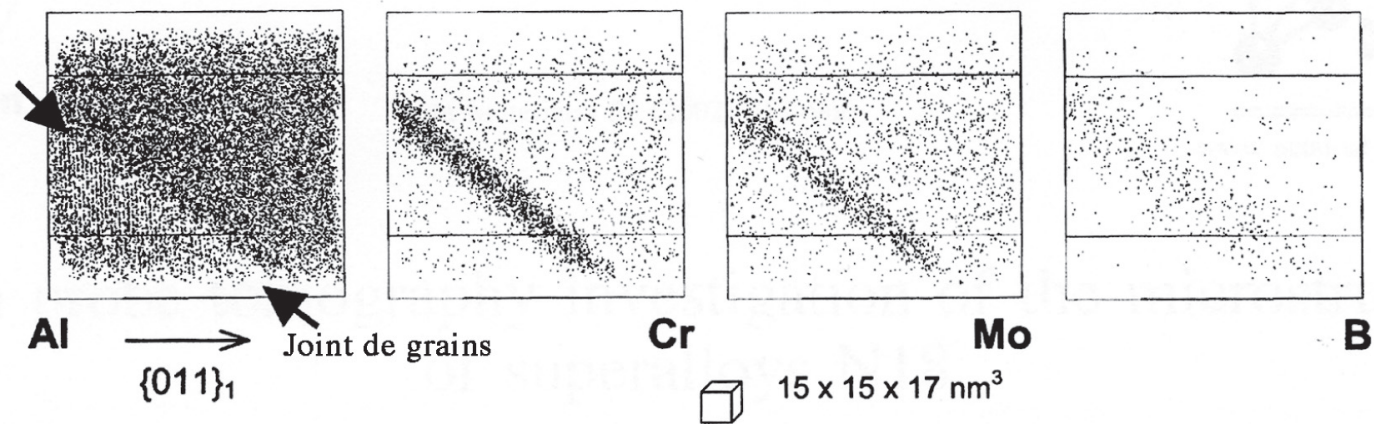

Figure 10. Images 3D obtenues en sonde atomique tomographique de la ségrégation du chrome, du molybdène et du bore à un joint de grains d'un superalliage N18 (chaque point correspond à un atome). Le cœur du joint est appauvri en aluminium [11].

La relation entre la ségrégation et le défaut de structure du joint est elle aussi réciproque : le défaut attire le soluté, celui-ci piégé sur la ligne de dislocation modifie la structure de cœur de cette dislocation.

\subsection{Ségrégation hors d'équilibre aux joints de grains}

Une ségrégation hors d'équilibre, dite encore cinétique, résulte généralement d'une interaction entre les atomes d'un des constituants d'une solution solide avec les lacunes thermiques en excès dans cette solution suite à une trempe ou à une grande vitesse de déformation et/ou avec les défauts interstitiels créés par irradiation [13]. Elle accompagne également la migration des joints de grains qui se produit lors de la restauration et de la recristallisation d'un matériau ou sous l'effet d'un champ électrique. Il y a alors traînage des solutés par les joints en mouvement. Les paramètres qui influent sur les mécanismes de ségrégation après trempe sont : (i) la température de recuit initialqui détermine le pourcentage de 

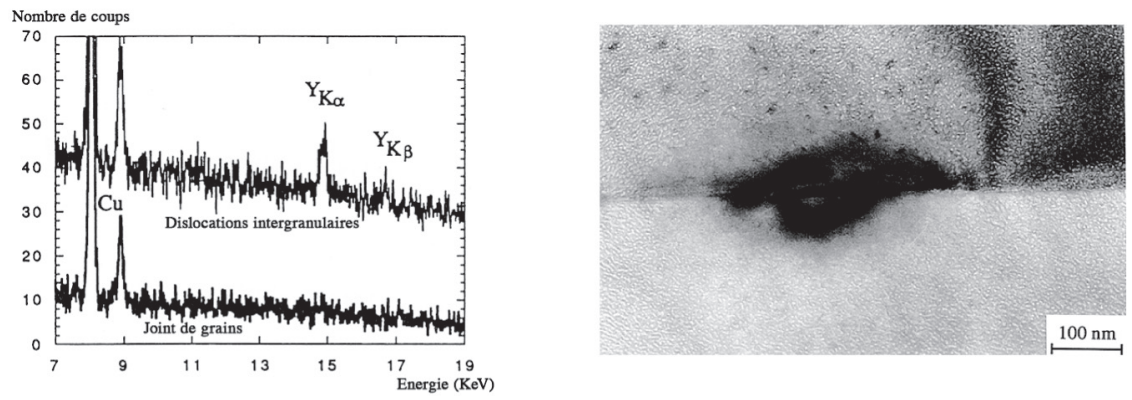

Figure 11. Spectres $X$ obtenus par STEM sur un joint de grains d'alumine dopée à l'yttrium : les pics d'yttrium sont associés à la présence de deux dislocations extrinsèques dans une région du joint de grains; ces dislocations sont observables par leur intense champ de déformation [12].

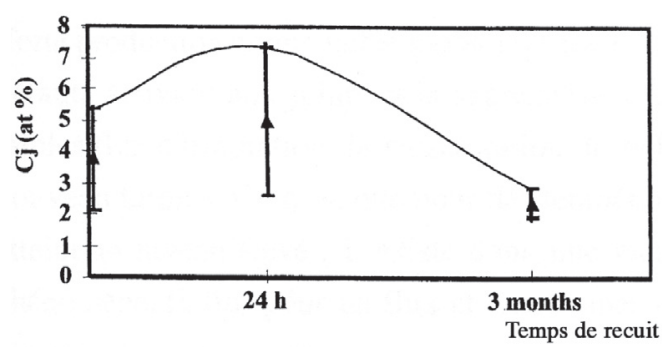

Figure 12. Influence du temps de maintien à $400^{\circ} \mathrm{C}$ sur la concentration moyenne en bore aux joints de grains d'un alliage Fe-40Al dopé avec 200 at.ppm de bore [14].

lacunes avant trempe, (ii) la vitesse de refroidissement qui permet à un nombre plus ou moins grand d'atomes d'atteindre la région intergranulaire, (iii) l'énergie d'interaction entre lacune et soluté formant le complexe et l'énergie d'activation de la diffusion de ce complexe.

La ségrégation hors d'équilibre est aussi caractérisée par une possibilité de "déségrégation" avec le temps résultant de la diffusion "retour" des espèces ségrégées dans leurs positions stables en matrice. Ainsi, dans le composé intermétallique $\mathrm{FeAl}$, après une forte ségrégation intergranulaire du bore due principalement à un mécanisme hors équilibre, la concentration aux joints de cet élément se fixe à une valeur faible correspondant à l'équilibre (Fig. 12) [14].

La ségrégation hors d'équilibre induite par irradiation diffère de celle résultant de traitements thermiques en ce sens que des complexes "interstitiel-soluté" se forment en addition aux complexes "lacunesoluté". Pour une espèce et une énergie données des particules incidentes, le niveau stationnaire de ségrégation induite dépend de leur flux (en déplacements par atome par seconde) et de la température d'irradiation. Ce n'est que pour des températures et des flux intermédiaires que la ségrégation atteint un niveau élevé : il existe donc une gamme de conditions d'irradiation donnant lieu à ce phénomène.

Qu'elle soit d'équilibre ou hors équilibre, la ségrégation intergranulaire constitue souvent un stade préliminaire à la précipitation. Il est parfois difficile de préciser la transition entre les deux phénomènes comme c'est le cas lorsqu'un film bidimensionnel de structure et de composition identiques à celles du composé à trois dimensions se forme dans l'interface.

\section{PRÉCIPITATION INTERGRANULAIRE}

Plusieurs raisons fondamentales expliquent une précipitation préférentielle aux joints de grains :

- Une première raison thermodynamique est la diminution de la barrière d'activation de la germination hétérogène par rapport à celle de germination homogène. 


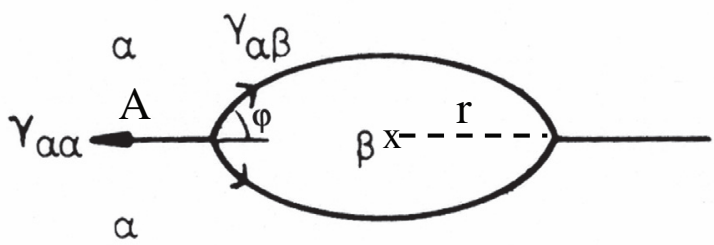

(a)

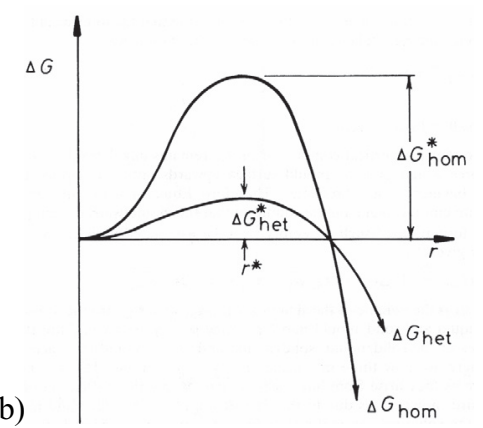

Figure 13. (a) Forme allotriomorphe d'un germe formé sur un joint de grains général ; (b) comparaison de l'énergie de germination hétérogène (maximum de la courbe) de ce germe par rapport à l'énergie de germination homogène d'un germe sphérique dans un cristal.

- Un coefficient de diffusion intergranulaire bien supérieur au coefficient de diffusion en volume permet aux atomes ségrégés de migrer rapidement vers tout germe critique de seconde phase dans un joint.

- Une forte ségrégation intergranulaire entraine une saturation du joint en atomes de solutés et conduit à la formation de précipités.

\subsection{Aspect thermodynamique}

La variation totale d'enthalpie libre $\Delta \mathrm{G}$ qui accompagne l'apparition d'un germe de seconde phase à un joint de grains s'écrit :

$$
\Delta \mathrm{G}=V \Delta \mathrm{G}_{\mathrm{V}}+\sum \mathrm{A}_{\alpha / \beta} \gamma_{\alpha / \beta}-\mathrm{A}_{\alpha / \alpha} \gamma_{\alpha / \alpha}
$$

$\Delta \mathrm{G}_{\mathrm{V}}$ est la variation, par unité de volume, de l'enthalpie libre de volume, $\mathrm{A}_{\alpha / \beta}$ est l'aire occupée par chacune des interfaces $\alpha / \beta$ et $\gamma_{\alpha / \beta}$ l'énergie de ces interfaces par unité de surface, $\mathrm{A}_{\alpha / \alpha} \gamma_{\alpha / \alpha}=\Delta \mathrm{G}_{\mathrm{D}}$, est l'énergie gagnée par relaxation du défaut que constitue la partie du joint de grains sur laquelle s'effectue la germination. Le premier terme et le troisième sont négatifs donc favorables à la germination, le second terme est positif puisqu'il correspond à la création de surface, il s'oppose au changement de phase. La germination a lieu lorsque le terme $\Delta \mathrm{G}_{\mathrm{T}}$ diminue avec l'augmentation de taille du germe. On montre que cette condition est réalisée pour un rayon critique d'un germe intergranulaire de forme "allotriomorphe" identique au rayon d'un germe sphérique qui se forme en matrice. Mais la barrière d'activation est considérablement diminuée, d'autant plus que l'angle de contact $\varphi$ est petit (Fig. 13).

L'équilibre des tensions interfaciales au point triple A nécessite que soit remplie la condition suivante :

$$
\cos \varphi=\frac{\gamma_{\alpha / \alpha}}{2 \gamma_{\alpha / \beta}}
$$

La tendance au dépôt du germe $\beta$ sur le support que constitue le joint de grains $\alpha / \alpha$ est d'autant plus forte que $\varphi$ tend vers 0 et donc que le coefficient de mouillage $\cos \varphi=\mathrm{m}$ tend vers 1 . L'efficacité d'un joint de grains comme site de germination hétérogène dépend donc des valeurs relatives de son énergie et de celles des interfaces susceptibles de se former entre la seconde phase et la phase mère. En réalité, un germe intergranulaire peut présenter diverses morphologies dépendant non seulement des énergies mais aussi de l'orientation du plan du joint dans l'un et l'autre des cristaux et de la présence de dislocations extrinsèques dont les champs de contraintes à grande distance peuvent attirer fortement les atomes de solutés. Il en résulte généralement une distribution non homogène des précipités intergranulaires d'un joint de grains à l'autre et le long d'un même joint de grains. 


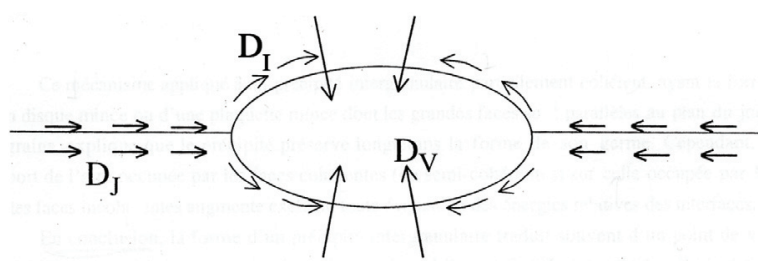

Figure 14. Processus de diffusion intervenant pour expliquer la croissance d'un germe à un joint de grains et qui opère dés le début de la stabilisation du germe [15].

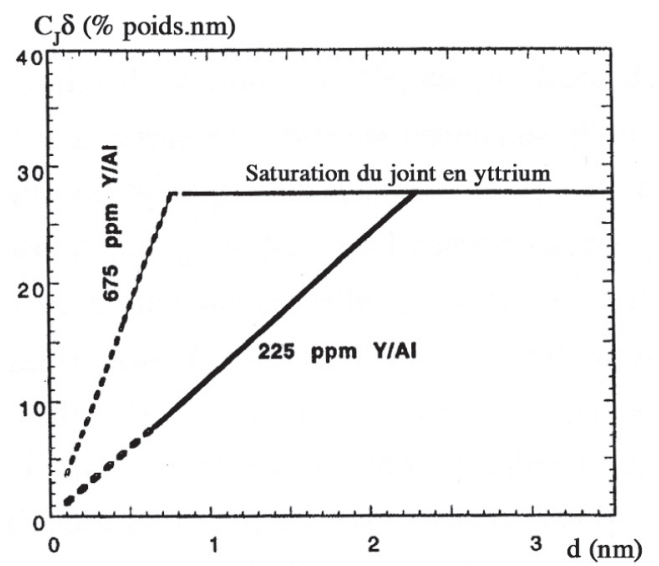

Figure 15. Évolution de la concentration d'yttrium aux joints de grains d'une alumine polycristalline. La saturation en yttrium correspond à l'apparition de précipités de grenats aux joints de grains, d'autant plus rapide que le taux de dopage en ytrrium est élevé [16].

\subsection{Aspect diffusionnel}

La croissance d'un précipité de forme allotriomorphe à un joint de grains dépend de trois processus de diffusion : relativement lent en volume, rapide au joint de grains et aux interfaces $\alpha / \beta$. C'est le mécanisme de la plaque collectrice d'Aaronson (Fig. 14) [15]. Il est logique de penser que cet ensemble de processus opère dès le début de la formation du germe.

Il est évident que la ségrégation préférentielle d'un élément aux joints de grains favorise l'apparition d'un composé défini comme suggéré par la formation de $\mathrm{Fe}_{3} \mathrm{P}$ au sein d'une unité structurale. Ce lien ségrégation/précipitation est bien mis en évidence dans un polycristal d'alumine dopée à l'yttrium. Une croissance des grains - donc une diminution du volume occupé par les régions intergranulaires entraîne une redistribution de l'yttrium ségrégé aux joints de grains. La teneur moyenne en yttrium augmente jusqu'à saturation, des particules de grenat (YAg) apparaissent alors aux joints de grains. La taille critique des grains pour laquelle cette transition se produit diminue avec la teneur globale en yttrium dans l'alumine (Fig. 15) [16].

\subsection{Forme prévisible des précipités selon l'énergie du joint de grains}

Pour que la condition d'équilibre (6) soit remplie à un joint de grains d'énergie élevée, la germination d'un précipité incohérent de forme allotriomorphe est favorisée. En revanche, un précipité cohérent ou partiellement cohérent doit se former préférentiellement sur un joint de grains d'énergie relativement 


\begin{tabular}{lll|l}
$\alpha_{\mathrm{CC}}$ & \multirow{3}{*}{$\beta_{\mathrm{CFC}} \quad\{111\}$} & $\{110\}$ \\
\hline$\alpha_{\mathrm{CC}}$ & & $\{111\}$ & $\{110\}$
\end{tabular}

Figure 16. Un précipité prend la forme d'un film mince étalé le long d'un joint de grains symétrique dont le plan est le plan d'habitat du précipité avec la matrice.

a)

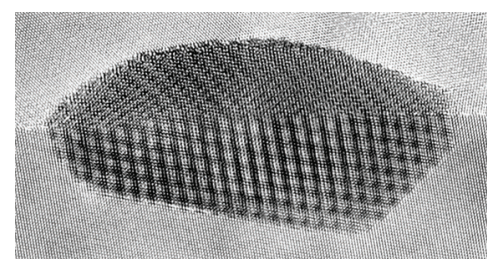

(b)

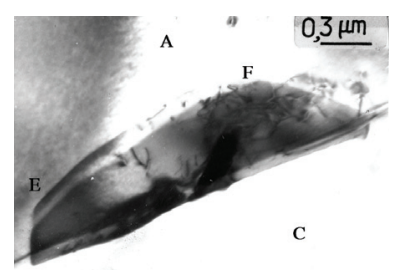

Figure 17. (a) Cristal de plomb au joint de grains d'un film mince d'aluminium présentant une forme semiallotriomorphe dans le grain supérieur et deux facettes dans le grain inférieur [18] ; (b) Précipité d'austénite à un joint de ferrite : la facette EF est parallèle à un plan d'habitat austénite/ferrite [19].

faible. Les interfaces cohérentes ou semi-cohérentes sont planes et confèrent au précipité une forme avec facettes. Plusieurs formes sont envisageables et observées selon le plan du joint de grains. Dans le cas le plus favorable, le plan du joint de faible énergie est un plan dense d'habitat entre précipité et matrice dans les deux cristaux, alors le germe de seconde phase s'étale comme un film des deux côtés du joint de grains (Fig. 16). Cette situation intervient entre une phase $\mathrm{CC}$ et une phase CFC, pour un joint de grains symétrique de plan $\{110\}$ si la phase mère est $C C$ et pour un joint de grains de plan $\{111\}$ si elle est CFC, pourvu que les distances interatomiques soient voisines. Si le joint est asymétrique, avec un plan $\{110\}_{\text {CC }}$ ou $\{111\}_{\text {CFC }}$ dans un cristal seulement, l'énergie du joint est plus élevée, un film de précipité avec une interface cohérente peut cependant s'étendre parallèlement au plan dense d'un seul côté du joint de grains.

En dehors de ces deux situations extrêmes, diverses morphologies de précipités existent à un joint de grains selon les énergies relatives des différentes interfaces $\alpha / \beta$ et du joint $\alpha / \alpha$ ainsi que de l'orientation d'une facette de faible énergie par rapport au plan du joint. Ces formes peuvent être déduites graphiquement sur la base de la construction de Wulff généralisée (voir Lee et Aaronson [17]). La figure 17 montre à deux échelles différentes la forme d'un précipité de seconde phase à un joint de grains. Une particule de plomb observée en METHR présente deux facettes cohérentes (ou semi-cohérentes) dans un des cristaux [18]. L'image en MET révèle qu'après grossissement d'un précipité d'austénite à un joint de ferrite, une facette parallèle à un plan d'habitat austénite/ferrite s'est maintenue dans un cristal [19].

\subsection{Croissance des précipités intergranulaires}

Un précipité, observé à l'échelle de la microscopie optique a rarement la forme originelle de son germe. La croissance entraîne la perte de cohérence de certaines interfaces et des interactions avec des obstacles (dislocations, solutés, autres précipités) modifient cette forme. Cependant, les mécanismes de croissance dépendant fortement des types d'interfaces qui bordent le précipité, on peut dans certains cas observer des formes de précipités intergranulaires qui ont hérité de la forme initiale du germe.

\subsubsection{Migration d'une interface courbe incohérente}

Pour qu'une interface incohérente migre, deux processus peuvent intervenir : le transfert des atomes au travers de l'interface et la redistribution du soluté de la matrice vers le précipité ou vice-versa. Le premier processus contrôle la croissance dans les tout premiers stades tandis que l'extension du 


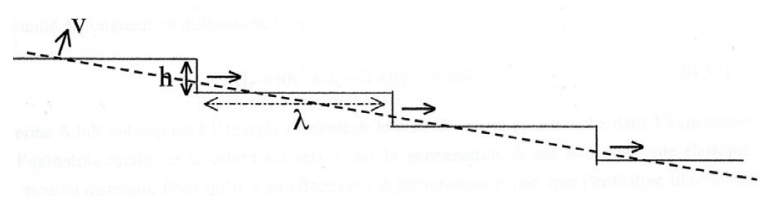

Figure 18. Schéma montrant que la migration d'une interface plane se produit par déplacement de marches nanométriques. Le plan du joint "moyen" (pointillé) se déplace lentement selon v.

(a)

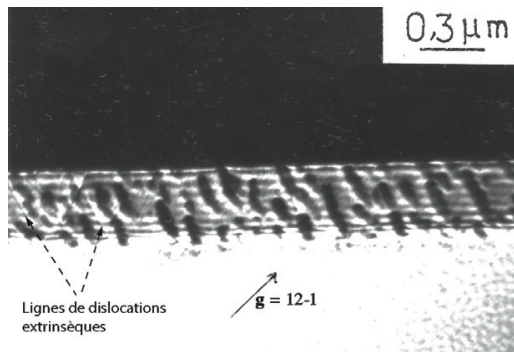

(b)

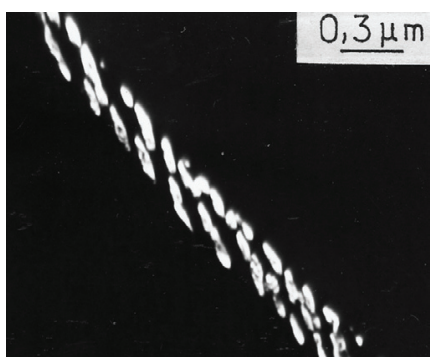

Figure 19. Images électroniques en champ sombre montrant des microprécipités de carbure de chrome $\mathrm{Cr}_{23} \mathrm{C}_{6}$ alignés parallèlement aux lignes de dislocations extrinsèques dans des joints de grains de l'alliage $\mathrm{Fe}-9 \% \mathrm{cr}-100$ ppm de Carbone : (a) joint vicinal à $2^{\circ}$ de $\Sigma 5$; (b) joint général [20].

précipité est gouvernée par la diffusion en volume. La vitesse de croissance est proportionnelle à (D/t) $1 / 2$ avec D le coefficient de diffusion du soluté dans la phase mère et $\mathrm{t}$ le temps de croissance. Les vitesses de croissance ont des ordres de grandeur très différents selon la sursaturation de la solution solide initiale en soluté et selon la température. Lorsqu'un germe allotriomorphe est situé à un joint de grains un mécanisme complexe appelé "mécanisme de la plaque collectrice" s'ajoute à la diffusion en volume vers le germe (Fig. 14) [15]. Le germe s'étire parallèlement au plan du joint plus qu'il ne s'épaissit.

\subsubsection{Migration d'une interface plane (cohérente ou semi-cohérente)}

Les faces cohérentes étant souvent des plans denses des cristaux, il est peu probable de greffer des atomes supplémentaires sur ces plans et le mécanisme de croissance par diffusion au travers de l'interface est peu probable. Les atomes de soluté s'adsorbent sur les marches, souvent présentes le long d'une interface en moyenne plane. La croissance se fait par déplacement latéral des marches (Fig. 18). La vitesse de déplacement des marches est proportionnelle au coefficient de diffusion en volume et à la sursaturation de la phase initiale en soluté. Elle est inversement proportionnelle à la hauteur $\mathrm{h}$ des marches et peut donc être relativement grande pour des marches nanométriques. Cependant, un déplacement latéral même rapide n'induit qu'un mouvement lent de l'interface dans son ensemble, perpendiculairement à elle-même. La vitesse globale ne dépend pas de la hauteur des marches, elle est inversement proportionnelle à leur longueur $\lambda$. Une interface sans marche est totalement immobile : c'est le cas d'une interface parfaitement cohérente. De plus, le nombre de marches ayant tendance à diminuer avec la croissance, celle-ci s'arrête s'il n'y a pas formation continue de nouvelles marches.

Ce mécanisme appliqué à un précipité intergranulaire partiellement cohérent, ayant la forme d'un disque mince ou d'une plaquette mince dont les grandes faces sont parallèles au plan du joint de grains, explique que le précipité préserve longtemps la forme de son germe. 

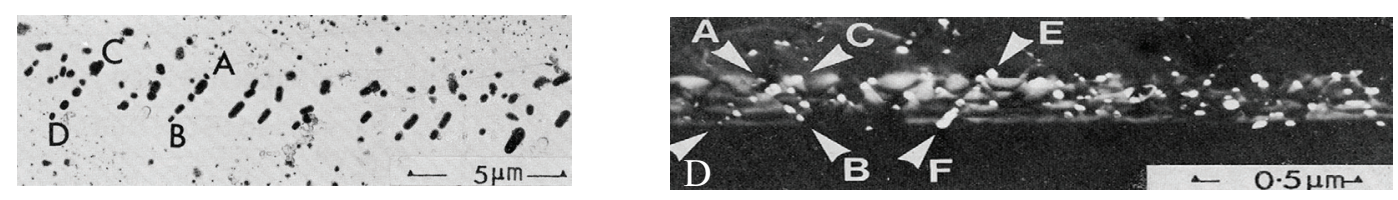

Figure 20. (a) Précipités intergranulaires de NbC observés sur une réplique extractive d'un échantillon d'acier austénitique. Les particules s'alignent et s'allongent suivant une direction préférentielle : (b) Image électronique en champ sombre montrant des particules de $\mathrm{NbC}$ associées à des dislocations extrinsèques de faible contraste (le long de $\mathrm{AB}, \mathrm{CD}$ et $\mathrm{EF}$ par exemple) [21].

\subsection{Précipitation intergranulaire et dislocations extrinsèques}

Les dislocations extrinsèques associées ou non à des marches sont des sites préférentiels de germination ; celle-ci est alors doublement "hétérogène" : par rapport au cristal et par rapport au joint de grains. La présence de précipités de carbures allongés selon les lignes de dislocations extrinsèques a été révélée par microscopie électronique en transmission dans le cas d'alliage fer-chrome (Fig. 19) [20] et d'acier inoxydable (Fig. 20) [21].

Pour conclure, rappelons que les précipités intergranulaires ont une influence notoire sur toutes les propriétés des joints de grains, ils constituent, entre autres, des freins à la migration des joints et sont des sites préférentiels de corrosion. Localisés sur les dislocations extrinsèques, ils épinglent celles-ci avec des répercussions sur tous les processus nécessitant le mouvement de ces dislocations soit dans le joint lui-même, soit du joint vers les cristaux. Aussi bien dans le cas de la ségrégation que dans celui de la précipitation, il y a couplage entre la chimie et les processus mécaniques aux joints de grains.

\section{Références}

[1] Mac Lean D., in "Grain Boundaries in metals" (Clarendon Press, Oxford, 1957).

[2] Seah M.P. and Hondros E.D., Proc. R. Soc. London A 335 (1973) 191.

[3] Lejcek P. and Hofmann S., Interface Science 1 (1993) 163.

[4] Bouchet D., Priester L., Scr. Metall. 20 (1986) 061 et 21 (1987) 475.

[5] Swiatnicki W., Lartigue Korinek S. and Laval J.Y., Acta Metall. Mater. 43 (1995) 795.

[6] Ference T.G. and Balluffi R.W., Scripta Metall. 22 (1988) 1929.

[7] Sickafus K. and Sass S.L., Scripta Metall. 18 (1984) 165.

[8] Rittner J.D. and Seidman D.N., Acta Mater. 45 (1997) 3191.

[9] Lezzar B., Khalfallah O., Larere A. and Paidar V. and Hardouin Duparc O., Acta Mater. 52 (2004) 2809.

[10] Hashimoto M., Ishida Y. and Yamamoto R. and Doyama M., Acta Metall. 32 (1984) 1.

[11] Cadel E., Lemarchand D., Chamberland S. and Blavette D., Acta Mater. 50 (2002) 957.

[12] Bouchet D., Dupau F. and Lartigue-Korinek S., Microsc. Microanal. Microstruct. 4 (1993) 561.

[13] Saindrenan G., Le Gall R. and Christien F., in " Endommagement interfacial des métaux Ségrégation interfaciale et conséquences”, Ellipses (2002) Paris.

[14] Frackiewicz A., Gay A.S. and Biscondi M., Mat.Sci. Eng. A 258 (1998) 108.

[15] Aaronson H.I. and Lee J.K., in "lectures on the theory of phase transformations", Ed. by H.I. Aaronson (1975) p.103

[16] Gruffel P. and Carry P., J. Euro. Ceram. Soc. 11 (1993) 189.

[17] Lee J.K. and Aaronson H.I., Acta Metall. 23 (1975) 799.

[18] Dahmen U. and Johnson E., Microscopy + Microanalysis, 5 (1999) 134.

[19] Priester L. "Les joints de grains - De la Thérie à l'Ingénierie”, EDP. Sciences, Les Ulis (2006).

[20] Lartigue S. Priester L., Acta Metall. 31 (1983) 1809.

[21] Jones A.R. and Howell P.R. and Ralph B., J. Mater. Sci. 11 (1976) 1593. 